\title{
Analysis of Skin, Hair and Nail Diseases in the Adults with Beta Thalassemia Major
}

\author{
Erişkin Beta Talasemi Major Hastalarında Eşlik Eden Deri, Saç ve Tırnak Bulgularının Araştırılması
}

\section{Suzan Demir Pektas ${ }^{1}$, Emine Tugba Alatas ${ }^{1}$, Gokhan Pektas ${ }^{2}$, Volkan Karakus ${ }^{2}$, Fatif Mehmet Azik ${ }^{3}$, Gursoy Dogan ${ }^{1}$}

${ }^{1}$ Department of Dermatology, Mugla Sitki Kocman University Faculty of Medicine, Mugla, Turkey

${ }^{2}$ Department of Hematology, Mugla Sitki Kocman University Faculty of Medicine, Training and Research Hospital, Mugla, Turkey

${ }^{3}$ Department of Pediatric Hematology, Mugla Sitki Kocman University Faculty of Medicine, Mugla, Turkey

\section{ABSTRACT}

Objective: $\beta$-thalassemia major (BTM) is a genetic disorder necessitating frequent blood transfusions, which is characterized by functional and physiological disorders in multiple organs. We aimed to research the prevalence of associated skin, hair, and nail disorders, and their relationship with clinical and laboratory features of the disease in adult BTM patients and healthy control groups.

Materials and Methods: We prospectively analyzed the prevalence of associated skin, hair, and nail disorders. We recorded demographic features, laboratory findings skin, hair, and nail disorders in the participants.

Results: Atotal of 71 BTM patients ( 36 males and 35 females) and 50 age and sex matched healthy individuals ( 36 females and 36 males) were included in the study. $32(45.1 \%)$ of the patients and $3(4.2 \%)$ of the control groups had hair, $26(36.6 \%)$ of the patients and $6(8.3 \%)$ of control groups had nail diseases. The most common skin findings xerosis were determined in 66 (93\%), hair findings, telogen effluvium were determined in $16(22.5 \%)$, and nail findings, dystrophic nail were determined $9(12.7 \%)$ in patients. BTM patients had significantly higher fruquent xerosis, scar, pigmentation disorders, nevus, pruritus, infections, nail and hair disorders than control groups. Average age of patients with pruritus and nevus were significantly enhanced. Postinflammatory hyperpigmentation and melanocytic nevus in male patients had more higher than female patients. Telogen effluvium in female patients was more higher than male patients. Ferritin levels of the patients with ephelide, diffuse bronze pigmentation (DBP) and telogen effluim had significantly enhanced.

Conclusion: Age, gender, medication history and ferritin level are important factors for the development of dermatological disorders among BTM patients.

Key Words: Beta thalassemia major, dermatological disease, hair, skin, nail

\section{ÖZET}

Amaç: $\beta$-talasemi majör (BTM), çoklu organlarda fonksiyonel ve fizyolojik bozukluklar ile karakterize, sık kan transfüzyonu gerektiren genetik bir hastalıktır. Yetişkin BTM hastalarında ve sağlıklı bireylerde kontrollerde cilt, saç ve tırnak hastalıklarının yaygınlıklarını ve bunların klinik ve laboratuvar özellikleriyle olan ilişkilerini araştırmayı amaçladık.

Materyal ve Yöntem: Ileriye dönük olarak, buna bağlı deri, saç ve tırnak hastalıklarının yaygınlığını analiz ettik. Katıımcıların demografik özellikleri, laboratuar bulguları, saç, tırnak ve saç bulgularını kaydettik.

Bulgular: Araștırmaya toplam 71 BTM hastası (36 erkek ve 35 kadın) ve 50 yas ve cinsiyet ile uygun sağıklı bireyler ( 36 kadın ve 36 erkek) dahil edildi. Hastaların 32'sinde (\%45.1) ve kontrol gruplarının 3'ünde (\%4.2) saç bulguları, hastaların 26'sında (\%36.6) ve kontrol gruplarının 6'sında (\%8.3) tırnak bulguları tespit edildi. En yaygın deri bulguları kserozis $66(\% 93)$, saç bulguları, telogen effluvium 16 (\% 22.5), tırnak bulguları, distrofik tırnak 9 (\% 12.7) hastada tespit edildi. BTM'li hastalarda, kontrol grubuna göre anlaml derecede yüksek sıklıkta kserozis, yara, pigmentasyon bozukluğu, nevüs, pruritus, enfeksiyon, tırnak ve saç hastalıkları vardı. Pruritus ve nevüs hastalarının ortalama yaş önemli derecede yüksekti. Erkek hastalarda postenflamatuvar hiperpigmentasyon ve melanositik nevüs, kadın hastalardan daha yüksekti. Kadın hastalarda telogen effluvium, erkek hastalardan daha yüksekti. Efelid, diffuse bronz pigmentasyon (DBP) ve telojen effluim bulunan hastaların ferritin düzeyleri anlamlı olarak yüksekti.

Sonuç: BTM'li hastalarda deri hastalıklarının gelişiminde yaş, cinsiyet, ilaç öyküsü ve ferritin düzeyi önemli faktörlerdir.

Anahtar Sözcükler: Beta talasemi major, dermatolojik hastalık, saç, cilt, tırnak 


\section{INTRODUCTION}

Thalassaemia is the most common monogenic hematologic hereditary disease in the World (1). In patients with beta thalassemia major (BTM); without transfusion and chelation treatment, life expectancy from the time of diagnosis is measured in months. In patients, iron accumulation develops in the tissues associated with continuous blood transfusions, low hepsin levels and ineffective erythropoiesis and consequently oxidative stress occurs (2). With this vicious circle, functional and physiological disorders occur in many organs such as skin, liver, heart, pancreas, gonad, pituitary and thyroid $(3,4)$. In the literature, there are few studies about the frequency of dermatological diseases in patients with $\operatorname{BTM}(2,4,5)$. Beside this, progressively when the increase of iron accumulation in the body is considered, as far as we know there is no further research about dermatological disorders in only adult BTM patients.

In our research, the aim was to investigate the relationship between the frequency of skin, hair and nail disorders associated with BTM patients and, clinical and laboratory characteristics of dermatological disorders.

\section{MATERIALS and METHODS}

This prospective, randomized study has been approved by the ethics committee of Mugla Sitki Kocman University. This study was practised between the dates May-November 2016, at Mugla Sitki Kocman University Training and Research Hospital Thalassemia Center, cross-sectionally in 71 adult BTM patients under regular follow-up and treatment and 72 healthy controls. Written consent form was obtained from all the partipicants included in the study. The data were obtained from patient follow-up files and the hospital automation system. Dermatological diseases were practised according to ICD-10 system. All partipicants's skin, hair and nails were examined by a dermatologist. Patients' age, gender, skin, hair and nail diseases, family history, thalassemia period, comorbid diseases, medications used and laboratory examination results (complete blood count, biochemical values and ferritin level) were recorded. Partipicants with a number of melanocytic nevus $>20$ were included in the study in terms of melanocytic nevus. Direct microscopic examination and/or culture and/or pathologic examination were performed on the patients who were seen as required, by taking samples from the lesion area (incisional biopsy, scraping and swab material). Statistics

To complete statistical assessments, the Statistical Package for Social Sciences (SPSS) software for Windows 22 (IBM SPSS Inc., Chicago, USA) was used. Quantitative data were shown as mean \pm standard deviation (SD) and qualitative data were shown as case number and percentile slice. A Pearson chi-squared or Fisher exact test was used to evaluate categorical variables. The distribution of the data was checked by the Kolmogorov Smirnov test. The mean differences of continuous variables with normal distribution between groups were compared with an independent sample student- t-test and for those with non-normal distribution were compared with the Mann-Whitney $U$ test when applicable. A $p$-value less than 0.05 was considered to be statistically significant.

\section{RESULTS}

Of the 71 patients included in the study, 35 were female, 36 were male and the average age was $29.6 \pm 8.3$ years and the 72 healthy control included in the study, 36 were female, 36 were male and the average age was $29.5 \pm 8.9$ years $(p>0.05)$. The comparison of demographic feature and laboratory findings of the participants are summarized in Table 1.

Table 1. Clinical and demographic characteristics of subjects

\begin{tabular}{|c|c|c|c|}
\hline Characteristic of partipicants & $\begin{array}{c}\text { Patients } \\
\text { (n:71) }\end{array}$ & Controls (n:72) & $\mathbf{P}$ \\
\hline Age(years) (mean $\pm S D)$ & $29.6 \pm 8.3$ & $29.54 \pm 8.965$ & \multirow{6}{*}{$\begin{array}{c}0.895^{*} \\
0.933^{* * * *} \\
<0.001^{* * *} \\
<0.001^{* * *} \\
* \\
<0.001^{* * *} \\
* \\
<0.001^{* * *} \\
* \\
\end{array}$} \\
\hline Gender, Male $n(\%)$ & $36(50.7)$ & $36(50)$ & \\
\hline Family history & $47(\% 66.2)$ & - & \\
\hline Hematocrit (\%) & $28.6 \pm 3.1$ & $40.2 \pm 2.4$ & \\
\hline Hemoglobin (gr/dl) & $9.0 \pm 0.9$ & $13.4 \pm 1.3$ & \\
\hline Ferritin $(\mathrm{ng} / \mathrm{mL})$ & $\begin{array}{c}1337.6 \pm 1298 \\
5\end{array}$ & $70.5 \pm 15.9$ & \\
\hline Comorbid conditions & $62(87.3 \%)$ & & \\
\hline Osteoporosis & $48(67.6 \%)$ & & \\
\hline Diabetes Mellitus & $18(25.4 \%)$ & & \\
\hline Hypothyroidism & $11(15.5 \%)$ & & \\
\hline Gastritis & $9(12.7 \%)$ & & \\
\hline Depression & $8(11.3 \%)$ & & \\
\hline Hyperlipidemia & $6(8.5 \%)$ & & \\
\hline Epilepsy & $5(7 \%)$ & & \\
\hline Hearing loss & $5(7 \%)$ & & \\
\hline Neurofibromatosis & $5(7 \%)$ & & \\
\hline Mental retardation & $4(5.6)$ & & \\
\hline Asthma & $4(5.6 \%)$ & & \\
\hline Hypogonadism & $3(4.2 \%)$ & & \\
\hline Chelator agents & $70(98.5 \%)$ & & \\
\hline Deferasirox & $56(78.9 \%)$ & & \\
\hline Deferiprone & $19(26.8 \%)$ & & \\
\hline Deferoxamine & $16(22.5 \%)$ & & \\
\hline \multicolumn{4}{|l|}{ Non-chelator agents } \\
\hline Osteoporosis treatment & $48(67.6 \%)$ & & \\
\hline Levothyroxine & $10(14.1 \%)$ & & \\
\hline Metformin & $8(11.3 \%)$ & & \\
\hline Insulin & $7(9.9 \%)$ & & \\
\hline Androgen & $6(8.5 \%)$ & & \\
\hline Proton pump inh. & $4(5.6 \%)$ & & \\
\hline Folic acid & $30(42.3 \%)$ & & \\
\hline Antiepileptic & $3(4.2 \%)$ & & \\
\hline Antidepressant & $1(1.41 \%)$ & & \\
\hline
\end{tabular}

*Student t-test, ${ }^{* *}$ fisher exact test, ${ }^{* * *}$ Pearson chi-square tests, ${ }^{* * *}$ Mann-Whitney $U$ $\mathrm{n}$; number of volunteer

Statistically significant associations were expressed as $(* p<0.05)$

Skin symptoms were detected in all of the patients and $6(8.3 \%)$ of controls. $32(45.1 \%)$ of the patients and $3(4.2 \%)$ of the controls and had hair and $26(36.6 \%)$ of the patients and $6(8.3 \%)$ of controls had nail diseases. The most common skin findings xerosis were determined in $66(93 \%)$, the most common hair findings telogen effluvium were determined in $16(22.5 \%)$, and the most common nail finding dystrophic nail were determined in $9(12.7 \%)$ patients. BTM patients had significantly higher frequency xerosis, scar, pigmentation disorders, nevus, pruritus, infections, nail and hair disorders than controls $(p<0.001$ for all). The comparison of skin, hair and nail findings of the participants are summarized in Table 2. 
Table 2. Comparation of dermatological diseases in patients with beta thalassemia major and control

\begin{tabular}{|c|c|c|c|}
\hline Dermatological diseases & Patients $\mathrm{n}(\%)$ & Control n(\%) & $\mathbf{P}$ \\
\hline Xerosis & $66(93.0 \%)$ & $6(8.3 \%)$ & $<0.001^{*}$ \\
\hline Pigmentation disease & $55(77.5 \%)$ & $9(12.5 \%)$ & $<0.001^{*}$ \\
\hline Ephelides & $45(63.4 \%)$ & $3(4.2 \%)$ & $<0.001^{* *}$ \\
\hline $\begin{array}{l}\text { Postinflammatory hyperpigmentation } \\
\text { Idiopathic guttate hypomelanosis }\end{array}$ & $\begin{array}{l}23(32.4 \%) \\
16(22.5 \%)\end{array}$ & $\begin{array}{l}5(6.9 \%) \\
0\end{array}$ & $\begin{array}{l}<0.001^{* *} \\
<0.001^{* *}\end{array}$ \\
\hline Melasma & $7(9.9 \%)$ & $4(5.6 \%)$ & $0.367^{* *}$ \\
\hline Diffuse bronze pigmentation & $4(5.6 \%)$ & 0 & $0.058^{* *}$ \\
\hline Akantozis Nigricans & $2(2.8 \%)$ & 0 & $0.245^{* *}$ \\
\hline Solar elastosis & $2(2.8 \%)$ & 0 & $0.245^{* *}$ \\
\hline Cafe au lait & $1(1.4 \%)$ & 0 & $0.497^{* *}$ \\
\hline Nevus & $40(56.3 \%)$ & $5(6.9 \%)$ & $<0.001 *$ \\
\hline Melanocytic nevus & $37(52.1 \%)$ & $3(4.2 \%)$ & $<0.001^{* *}$ \\
\hline Dermal nevus & $4(5.6 \%)$ & $1(1.4 \%)$ & $0.209 * *$ \\
\hline Depigmented nevus & $2(2.8 \%)$ & 0 & $0.245^{* *}$ \\
\hline Nevus spilus & $2(2.8 \%)$ & 0 & $0.245^{* *}$ \\
\hline Hemangioma & $1(1.4 \%)$ & $1(1.4)$ & $>0.999 * *$ \\
\hline Pruritus & $24(33.8 \%)$ & $4(5.6 \%)$ & $<0.001^{*}$ \\
\hline Hair diseases & $32(45.1 \%)$ & $3(4.2 \%)$ & $<0.001^{*}$ \\
\hline Telogen effluvium & $16(22.5 \%)$ & $1(1.4 \%)$ & $<0.001^{* *}$ \\
\hline Early whitening & $10(14.1 \%)$ & $1(1.4 \%)$ & $0.004^{* *}$ \\
\hline Alopecia areata & $3(4.2 \%)$ & 0 & $0.120 * *$ \\
\hline Androgenetic alopecia & $2(2.8 \%)$ & $1(1.4 \%)$ & $0.620 * *$ \\
\hline Poliosis & $1(1.4 \%)$ & 0 & $0.497^{* *}$ \\
\hline Nail diseases & $26(36.6 \%)$ & $6(8.3 \%)$ & $<0.001^{*}$ \\
\hline Dystrophic nail & $9(12.7 \%)$ & 0 & $0.001 * *$ \\
\hline Onycholysis & $6(8.5 \%)$ & $1(1.4 \%)$ & $0.063^{* *}$ \\
\hline Leukonychia & $4(5.6 \%)$ & 0 & $0.058^{* *}$ \\
\hline Koilonychia & $3(4.2 \%)$ & 0 & $0.120 * *$ \\
\hline Hemorrhagic nail & $3(3.6 \%)$ & 0 & $0.245^{* *}$ \\
\hline Onychophagia & $2(2.8 \%)$ & 0 & $0.497 * *$ \\
\hline Dermatoses & $24(33.8 \%)$ & $8(15.5 \%)$ & $0.788^{*}$ \\
\hline Keratosis pilaris & $8(11.3 \%)$ & $1(7.0 \%)$ & $0.017^{* *}$ \\
\hline Lichen simplex chronicus & $4(5.6 \%)$ & 0 & $0.058 * *$ \\
\hline Acne vulgaris & 0 & $3(4.2 \%)$ & $0.245^{* *}$ \\
\hline Irritant contact dermatitis & $3(4.2 \%)$ & $1(1.4 \%)$ & $0.366^{* *}$ \\
\hline Chronic urticaria & $3(4.2 \%)$ & $1(1.4 \%)$ & $0.366 * *$ \\
\hline Local hyperhidrosis & $3(4.2 \%)$ & $1(1.4 \%)$ & $0.366 * *$ \\
\hline Pitriazis alba & $3(4.2 \%)$ & $2(2.8 \%)$ & $0.681^{* *}$ \\
\hline Seborrheic dermatitis & $2(2.8 \%)$ & 0 & $0.245^{* *}$ \\
\hline Enfeksiyonlar & $20(28.2 \%)$ & $6(8.3 \%)$ & $<0.001^{*}$ \\
\hline Tinea pedis & $5(7 \%)$ & $1(1.4 \%)$ & $0.116 * *$ \\
\hline Folliculitis & $4(5.6 \%)$ & 0 & $0.058 * *$ \\
\hline Tinea versicolor & $3(4.2 \%)$ & 0 & $0.120 * *$ \\
\hline Herpes labialis & $3(4.2 \%)$ & $1(1.4 \%)$ & $0.366^{* *}$ \\
\hline Tinea cruris & $1(1.4 \%)$ & 0 & $0.497 * *$ \\
\hline Cellulitis & $1(1.4 \%)$ & 0 & $0.497^{* *}$ \\
\hline İmpetigo & $1(1.4 \%)$ & $1(1.4 \%)$ & $>0.999 * *$ \\
\hline Verruka vulgaris & $1(1.4 \%)$ & 0 & $0.497^{* *}$ \\
\hline Onychomycosis & $1(1.4 \%)$ & 0 & $0.620 * *$ \\
\hline Şelasyon tedavisine bağlı yan etkiler & $15(21.1 \%)$ & & \\
\hline Deferoxamine related atrophic scars & $6(8.5 \%)$ & & \\
\hline Deferoxamine-related urticaria & $7(9.8 \%)$ & & \\
\hline Deferiprone related erythema & $1(1.4 \%)$ & & \\
\hline Deferoxamine related erythema & $1(1.4 \%)$ & & \\
\hline
\end{tabular}

When dermatological diseases of the patients were compared with age, average age of patients with pruritus and nevus were more higher than patients with other dermatological disease (respectively, 33.7 \pm 8.7 years vs. $27.5 \pm 7.4$ years, $p=0.002$ and $31.4 \pm 8.8$ years vs. $27.4 \pm 7.3$ years, $p=0.046$ ). The comparison in age of skin, hair and nail findings of the participants are summarized in Table 3.

Table 3. Relationship between age of patients and accompanying dermatological diseases in patients with beta thalassemia major

\begin{tabular}{|c|c|c|c|}
\hline & \multicolumn{2}{|l|}{ Age } & \multirow[t]{2}{*}{ p } \\
\hline & $\begin{array}{l}\text { Present } \\
(\text { mean } \pm S D)\end{array}$ & $\begin{array}{l}\text { Absent } \\
(\text { mean } \pm S D)\end{array}$ & \\
\hline Xerosis & $29.7 \pm 8.4$ & $28.8 \pm 8.5$ & 0.821 \\
\hline Pigmentation disease & $29.3 \pm 7.6$ & $30.7 \pm 10.7$ & 0.564 \\
\hline Nevus & $31.4 \pm 8.8$ & $27.4 \pm 7.3$ & 0.046 \\
\hline Pruritus & $33.7 \pm 8.7$ & $27.5 \pm 7.4$ & 0.002 \\
\hline Hair diseases & $31.8 \pm 8.5$ & $27.9 \pm 7.9$ & 0.050 \\
\hline Nail diseases & $30.5 \pm 7.2$ & $29.1 \pm 8.9$ & 0.502 \\
\hline Dermatoses & $29.7 \pm 8.4$ & $29.6 \pm 8.47$ & 0.949 \\
\hline Infections & $28.9 \pm 6.3$ & $29.9 \pm 9.0$ & 0.651 \\
\hline $\begin{array}{l}\text { Side effects of chelator } \\
\text { agent }\end{array}$ & $32.4 \pm 10.2$ & $28.9 \pm 7.7$ & 0.146 \\
\hline
\end{tabular}

When dermatological diseases of the patients were compared with genders, the frequency of postinflammatory hyperpigmentation and melanocytic nevus in male patients had more frequency than female patients (in order; $22.5 \%$ vs. $9.8 \%, p=0.028$ and $32.4 \%$ vs. $19.7 \%$, $\mathrm{p}=0.044)$. The frequency of telogen effluvium in female patients was more higher than male patients $(16.9 \%$ vs. $5.6 \%, p=0.019)$. When the relationships between dermatological diseases and comorbid conditions of patients were examined, there were no statistically significant differences in all parameters between the group with comorbid conditionsand without comorbid conditions. When the relationship between labaratory values of patients and dermatological diseases were examined, ferritin levels of the patients with ephelide, diffuse bronze pigmentation (DBP) and telogen effluvium had more higher than patients without ephelides, DBP and telogen effluvium (in order; $1550.0 \pm 1147.9 \mathrm{ng} / \mathrm{mL}$ vs. $1384.6 \pm 1321.4 \mathrm{ng} / \mathrm{mL}, \mathrm{p}=0.012$ and $1516.0 \pm 1497.6$ vs. $1028.8 \pm 783.5, p=0.041$ and $1459.7 \pm 1396.8 \mathrm{ng} / \mathrm{mL}$ vs. $917.9 \pm 772.5 \mathrm{ng} / \mathrm{mL}, \mathrm{p}=0.023)$. The comparison in laboratory values of skin, hair and nail findings of the participants are summarized in Table 4. 
Table 4. Correlations of dermatological diseases and hemoglobin, hematocrit and ferritin levels in beta major thalassemia patients.

\begin{tabular}{|c|c|c|c|c|c|c|c|c|c|}
\hline \multirow[b]{2}{*}{ Dermatological diseases } & \multicolumn{2}{|c|}{ Hemoglobin (gr/dl) } & \multicolumn{4}{|c|}{ Hematocrite (\%) } & \multicolumn{3}{|l|}{ Ferritin $(\mathrm{ng} / \mathrm{mL})$} \\
\hline & $\begin{array}{l}\text { Present } \\
\text { (ort } \pm s s)\end{array}$ & $\begin{array}{l}\text { Absent } \\
\text { (ort } \pm s s)\end{array}$ & $\mathbf{p}$ & $\begin{array}{l}\text { Present } \\
\text { (ort } \pm s s)\end{array}$ & $\begin{array}{l}\text { Absent } \\
\text { (ort } \pm s s)\end{array}$ & $\mathbf{p}$ & $\begin{array}{l}\text { Present } \\
\text { (ort } \pm s s)\end{array}$ & $\begin{array}{l}\text { Absent } \\
\text { (ort } \pm s s) p\end{array}$ & \\
\hline Xerosis & $9.02 \pm 0.9$ & $9.2 \pm 0.6$ & 0.594 & $28.5 \pm 3.1$ & $30.2 \pm 4.2$ & 0.429 & $1358.6 \pm 1330.5$ & $1060.4 \pm 785.2$ & 0.678 \\
\hline Pigmentation disease & $9 \pm 1$ & $9.1 \pm 0.8$ & 0.702 & $28.7 \pm 3.3$ & $28.2 \pm 2.5$ & 0.580 & $1431.9 \pm 1392.6$ & $1013.6 \pm 858.4$ & 0.188 \\
\hline Ephelides & $9.2 \pm 0.9$ & $8.8 \pm 1.0$ & 0.158 & $29.0 \pm 3.0$ & $27.9 \pm 3.3$ & 0.124 & $1516.0 \pm 1497.6$ & $1028.8 \pm 783.5$ & $0.041^{*}$ \\
\hline Postinflammatory hyperpigmentation & $8.8 \pm 0.9$ & $9.1 \pm 0.9$ & 0.101 & $28.3 \pm 2.9$ & $28.7 \pm 3.3$ & 0.697 & $1156.4 \pm 844.7$ & $1424.5 \pm 1266.9$ & 0.971 \\
\hline Idiopathic guttate hypomelanosis & $8.6 \pm 1.0$ & $9.2 \pm 0.9$ & 0.050 & $27.9 \pm 3.6$ & $28.8 \pm 3.0$ & 0.256 & $898.5 \pm 515.1$ & $1465.4 \pm 1427.1$ & 0.058 \\
\hline Melasma & $8.7 \pm 1.2$ & $9.1 \pm 0.9$ & 0.321 & $28.1 \pm 4.3$ & $28.6 \pm 3.0$ & 0.756 & $1106.9 \pm 589.3$ & $1362.9 \pm 1353.7$ & 0.962 \\
\hline Diffuse bronze pigmentation & $8.0 \pm 0.9$ & $9.1 \pm 0.9$ & $0.021^{*}$ & $25.5 \pm 2.1$ & $28.8 \pm 3.1$ & $0.027^{*}$ & 1550. $0 \pm 1147.9$ & $1384.6 \pm 1321.4$ & $0.012^{*}$ \\
\hline Akantozis Nigricans & $8.5 \pm 0.7$ & $9.1 \pm 0.9$ & 0.407 & $28.0 \pm 1.4$ & $28.6 \pm 3.2$ & 0.798 & $3807.0 \pm 3698.2$ & $1266.0 \pm 1161.4$ & 0.126 \\
\hline Solar elastosis & $8.9 \pm 0.1$ & $9.0 \pm 0.9$ & 0.608 & $27.5 \pm 0.7$ & $28.6 \pm 3.2$ & 0.565 & $540.0 \pm 183.9$ & $1360.7 \pm 1309.5$ & 0.072 \\
\hline Cafe au lait & 10.0 & $9.0 \pm 09$ & 0.310 & 33 & $28.5 \pm 3.1$ & 0.169 & 861 & $1344.4 \pm 1306.1$ & $>0.999$ \\
\hline Nevus & $9 \pm 0,8$ & $9,1 \pm 1$ & 0.730 & $28,3 \pm 3$ & $28,9 \pm 3,4$ & 0.480 & $1052,6 \pm 681,4$ & $1705,4 \pm 1554,9$ & 0.363 \\
\hline Melanocytic nevus & $9.0 \pm 0.9$ & $9.1 \pm 1.0$ & 0.740 & $28.3 \pm 3.1$ & $28.9 \pm 3.2$ & 0.570 & $1006.2 \pm 589.0$ & $1698.3 \pm 1514.2$ & 0.238 \\
\hline Dermal nevus & $9.2 \pm 0.9$ & $9.0 \pm 0.9$ & 0.674 & $30.3 \pm 1.3$ & $28.5 \pm 3.2$ & 0.104 & $929.5 \pm 286.0$ & $1362.0 \pm 1331.4$ & 0.990 \\
\hline Depigmented nevus & $9.2 \pm 0.2$ & $9.0 \pm 0.9$ & 0.724 & $32.5 \pm 5.0$ & $28.5 \pm 3.1$ & 0.181 & $791.5 \pm 12.0$ & $1353.5 \pm 1313.6$ & 0.850 \\
\hline Nevus spilus & $8.6 \pm 0.8$ & $9.1 \pm 0.9$ & 0.587 & $28.0 \pm 2.8$ & $28.6 \pm 3.2$ & 0.876 & $532.5 \pm 219.9$ & $1360.9 \pm 1309.3$ & 0.089 \\
\hline Hemangioma & 8.9 & $9.0 \pm 0.9$ & 0.676 & 28.0 & $28.6 \pm 3.2$ & 0.901 & 772.0 & $1345.7 \pm 1305.6$ & 0.873 \\
\hline Pruritus & $8,8 \pm 1,1$ & $9,2 \pm 0,8$ & 0,357 & $28,1 \pm 2,8$ & $28,8 \pm 3,3$ & 0,633 & $1536,8 \pm 1445,2$ & $1235,9 \pm 1220$ & 0,697 \\
\hline Dermatoses & $9,1 \pm 0,9$ & $9 \pm 1$ & 0.787 & $28,5 \pm 2,7$ & $28,6 \pm 3,4$ & 0.864 & $1408,4 \pm 1324,1$ & $1301,5 \pm 1297,5$ & 0.631 \\
\hline Keratosis pilaris & $9.3 \pm 1.0$ & $9.0 \pm 0.9$ & 0.091 & & $28.5 \pm 3.1$ & 0.305 & $1243.6 \pm 613.7$ & $1349.6 \pm 1303.3$ & 0.373 \\
\hline Lichen simplex chronicus & $8.9 \pm 1.1$ & $9.0 \pm 0.9$ & 0.857 & & $28.6 \pm 3.2$ & 0.952 & $2860.3 \pm 2711.5$ & $1246.7 \pm 1141.9$ & 0.302 \\
\hline Irritant contact dermatitis & $9.4 \pm 0.8$ & $9.0 \pm 09$ & 0.577 & $29.4 \pm 3.3$ & $28.6 \pm 3.1$ & 0.904 & $1389.7 \pm 1150.4$ & $1335.3 \pm 1311.8$ & 0.925 \\
\hline Chronic urticaria & $9.1 \pm 0.9$ & $9.0 \pm 0.9$ & 0.840 & $\begin{array}{l}28.3 \pm 2.1 \\
29.0 \pm 3.6\end{array}$ & $28.6 \pm 3.1$ & 0.675 & $1292.3 \pm 810.9$ & $1339.6 \pm 1319.3$ & 0.777 \\
\hline Seborrheic dermatitis & $8.9 \pm 0.1$ & $9.0 \pm 0.9$ & 0.677 & $\begin{array}{l}28.0 \pm 3.6 \\
27.0 \pm 1.4\end{array}$ & $28.6 \pm 3.2$ & 0.426 & $817.5 \pm 116.7$ & $1352.7 \pm 1313.8$ & 0.824 \\
\hline Local hyperhidrosis & $8.3 \pm 0.6$ & $9.1 \pm 0.9$ & 0.157 & $27.3 \pm 1.2$ & $28.6 \pm 3.2$ & 0.434 & $677.0 \pm 279.7$ & $1366.8 \pm 1318.2$ & 0.194 \\
\hline Infections & $8,8 \pm 1$ & $9,1 \pm 0,9$ & 0.210 & $28,1 \pm 2,7$ & $28,7 \pm 3,3$ & 0.516 & $1192,5 \pm 946,2$ & $1394,5 \pm 1216,7$ & 0.632 \\
\hline Tinea pedis & $7.9 \pm 0.8$ & $9.1 \pm 0.9$ & $0.003^{*}$ & $26.6 \pm 2.5$ & $28.7 \pm 3.1$ & 0.139 & $946.2 \pm 661.0$ & $1367.3 \pm 1332.3$ & 0.362 \\
\hline Folliculitis & $8.9 \pm 1.1$ & $9.0 \pm 0.9$ & 0.819 & $28.0 \pm 3.4$ & $28.6 \pm 3.1$ & 0.764 & $843.8 \pm 252.5$ & $1367.1 \pm 1329.8$ & 0.801 \\
\hline Tinea versicolor & $9.3 \pm 1.2$ & $9.0 \pm 0.9$ & 0.503 & $29.0 \pm 3.6$ & $28.6 \pm 3.1$ & 0.904 & $792.0 \pm 249.8$ & $1361.7 \pm 1320.8$ & 0.577 \\
\hline Herpes labialis & $9.2 \pm 0.4$ & $9.0 \pm 0.9$ & 0.675 & $28.0 \pm 3.0$ & $28.6 \pm 3.2$ & 0.840 & $1598.7 \pm 1152.0$ & $1326.1 \pm 1310.6$ & 0.451 \\
\hline Tinea cruris & 10.0 & $9.0 \pm 0.9$ & 0.310 & 32.0 & $28.5 \pm 3.1$ & 0.282 & 413.0 & $1350.8 \pm 1302.6$ & 0.169 \\
\hline Cellulitis & 8.9 & $9.0 \pm 0.9$ & 0.761 & 28.0 & $28.6 \pm 3.2$ & 0.901 & 735.0 & $1346.2 \pm 1305.4$ & 0.732 \\
\hline Impetigo & 7.9 & $9.1 \pm 0.9$ & 0.113 & 28.0 & $28.6 \pm 3.2$ & 0.901 & 3204.0 & $1311.0 \pm 1287$ & 0.169 \\
\hline Verruka vulgaris & 10.0 & $9.0 \pm 0.9$ & 0.310 & 30.0 & $28.6 \pm 3.1$ & 0.535 & 3521.0 & $1306.4 \pm 1280.3$ & 0.113 \\
\hline onikomikoz & 8.9 & $9.0 \pm 0.9$ & 0.676 & 28.0 & $28.6 \pm 3.2$ & 0.901 & 699.0 & $1346.7 \pm 1305.1$ & 0.620 \\
\hline Side effects of chelator agent etkiler & $9.0 \pm 1.0$ & $9 \pm 0,9$ & 0.589 & $28,6 \pm 3,1$ & $28,6 \pm 3,2$ & 0.630 & $1888,1 \pm 1236,7$ & $1190,2 \pm 875,3$ & 0.317 \\
\hline Deferoxamine related erythema & 9,0 & $9,0 \pm 0,9$ & 0.986 & 25,0 & $28.6 \pm 3.1$ & 0.225 & 700.0 & $1346.7 \pm 1305.1$ & 0.676 \\
\hline Deferoxamine-related urticaria & $8.9 \pm 1.2$ & $9.1 \pm 0.9$ & 0.946 & 29.33 .2 & $28.5 \pm 3.2$ & 0.327 & $2142.9 \pm 2017.3$ & $1249.6 \pm 1185.2$ & $0.039 *$ \\
\hline Deferoxamine related atrophic scars & $9.0 \pm 0.8$ & $9.1 \pm 0.9$ & 0.832 & $28.1 \pm 3.2$ & $28.6 \pm 3.2$ & 0.912 & $1948.8 \pm 1926.2$ & $1281.2 \pm 1065.7$ & 0.565 \\
\hline Deferiprone related erythema & 10.3 & $9.0 \pm 0.9$ & 0.114 & 30.0 & $28.5 \pm 3.2$ & 0.543 & 928.0 & $1349.6 \pm 1315.0$ & 0.886 \\
\hline Hair diseases & $8,8 \pm 1$ & $9,2 \pm 0,8$ & 0.258 & $27,9 \pm 3$ & $29,1 \pm 3,2$ & 0.125 & $1151,9 \pm 1147,7$ & $1490 \pm 1405,8$ & 0.277 \\
\hline Telogen effluvium & $8.9 \pm 1.0$ & $9.1 \pm 0.9$ & 0.934 & $28.0 \pm 2.7$ & $28.7 \pm 3.3$ & 0.734 & $917.9 \pm 772.5$ & $1459.7 \pm 1396.8$ & $0.023^{*}$ \\
\hline Early whitening & $8.5 \pm 1.0$ & $9.1 \pm 0.9$ & 0.137 & $26.3 \pm 2.3$ & $28.9 \pm 3.1$ & $0.009 *$ & $1018.0 \pm 554.5$ & $1390.0 \pm 1378.3$ & 0.882 \\
\hline Alopecia areata & $9.0 \pm 1.0$ & $9.0 \pm 0.9$ & 0.989 & $27.7 \pm 3.1$ & $28.6 \pm 3.2$ & 0.635 & $2996.0 \pm 2968.2$ & $1264.5 \pm 1170.0$ & 0.060 \\
\hline Androgenetic alopecia & $9.5 \pm 0.7$ & $9.0 \pm 0.9$ & 0.444 & $34.0 \pm 2.8$ & $28.4 \pm 3.0$ & 0.024 & $1068.9 \pm 379.7$ & $1345.4 \pm 1315.4$ & 0.654 \\
\hline Poliosis & 8.0 & $9.0 \pm 0.9$ & 0.310 & 29.0 & $28.4 \pm 3.2$ & 0.817 & 870.0 & $1344.3 \pm 1306.2$ & 0.986 \\
\hline Nail diseases & $8,9 \pm 0,8$ & $9.0 \pm 0.9$ & 0.437 & $28,3 \pm 2,7$ & $28,7 \pm 3,4$ & 0.773 & $946,3 \pm 535,2$ & $1563,7 \pm 1541,1$ & 0.174 \\
\hline Dystrophic nail & $8.7 \pm 1.0$ & $9.0 \pm 0.9$ & 0.350 & $27.6 \pm 3.3$ & $28.7 \pm 3.1$ & 0.455 & $1096.8 \pm 720.4$ & $1372.6 \pm 1362.2$ & 0.849 \\
\hline Onycholysis & $9.3 \pm 0.8$ & $9.0 \pm 0.9$ & 0.448 & $\begin{array}{l}30.0 \pm 3.1 \\
6\end{array}$ & $28.4 \pm 3.1$ & 0.258 & $1030.8 \pm 583.0$ & $1365.9 \pm 1344.1$ & 0.817 \\
\hline Leukonychıa & $8.8 \pm 0.5$ & $9.1 \pm 1.0$ & 0.523 & $28.4 \pm 1.1$ & $28.6 \pm 3.2$ & $>0.999$ & $795.5 \pm 343.8$ & $1370.0 \pm 1327.7$ & 0.507 \\
\hline Onychophagia & 9.8 & $9.0 \pm 0.9$ & 0.479 & 30.0 & $28.5 \pm 3.2$ & 0.535 & 737.0 & $1346.2 \pm 1305.4$ & 0.789 \\
\hline Hemorrhagic nail & $8.7 \pm 0.5$ & $9.0 \pm 0.9$ & 0.544 & $25.5 \pm 0.7$ & $28.7 \pm 3.1$ & 0.097 & $730.0 \pm 381.8$ & $1355.2 \pm 1311.9$ & 0.483 \\
\hline Koilonychia & $8.9 \pm 0.8$ & $9.0 \pm 0.9$ & 0.777 & $28.3 \pm 1.2$ & $28.6 \pm 3.2$ & 0.989 & $823.7 \pm 337.6$ & $1360.3 \pm 1320.8$ & 0.596 \\
\hline
\end{tabular}


BTM is a hypochromic micrositeran anemia with multiple comorbid conditions and high morbidity and mortality rates (4). In addition to the initiation of treatment of the disease in pediatric age, with the recent developments, the disease can be followed long-term without any mortality in adulthood. In addition to disease-related effects in BTM, dermatologic side effects associated with chelation treatment agents are frequently encountered $(2,4)$. Research on the frequency of dermatologic diseases in BTM is limited and few in the literature $(2,4$, $5)$. In our study, skin diseases were determined in all, hair diseases were determined in 32, and nail diseases were determined in 26 patients. The most common skin finding was xerosis, hair finding was telogen effluvium, nail finding was dystrophic nail. Postinflammatory hyperpigmentation and melanocytic nevus in male BTM patients and telogen efflux in female BTM patients were more frequent. Age of patients with pruritus and nevus were more higher than patients with other dermatological disease. Ferritin levels of the patients with ephelide, DBP, deferoxamine-induced urticaria and telogen effluvium had more higher than patients without ephelides, DBP and telogen effluvium. There was decreased $\mathrm{Hb}$ value in patients with DBP and tinea pedis and Htc value in patients withDBP, early whitening and androgenetic alopecia.

In BTM, high iron accumulation occurs in the body with the increase in intestinal iron absorption due to chronic anemia, the inability to use iron as a result of ineffective erythropoiesis and transfusions (6). Free iron hydrogen peroxides can damage tissues by catalyzing the conversion to free radicals released into cellular membranes, proteins and DNA. In the researches, it was stated that iron has been implicated in the formation of hydroxyl radicals in various skin diseases including pigmentation disorders, inflammation and cancers and might show toxic effect $(7,8)$. Ferritin, on the other hand, is the main protective mechanism that links iron in various tissues. Ferritin, which holds iron soluble and non-toxic, inhibits the formation of reactive oxygen species those come up by iron's catalyst effect (9). Pourzand and friends reported that exposure of human primary skin fibroblasts to UVA radiation of FEK4 caused release of "free" iron in cells via ferritin proteolysis and this condition to UVA may be an important factor in causing deep damage $(10,11)$. To release free iron in skin cells with ferritin proteolysis and UV light, high temperature and other exogen stress factors like inflamation; is thought to have a pathogenic role in some dermatological diseases (11). Iron overload in BTM patients has been shown to cause oxidative damage to tissues by increasing free radical (12).

In the literature, there are few studies on dermatological disorders in BTM patients $(2,4,5)$. Dogramaci et all (4) stated that dermatological disorders were detected in $65(83 \%)$ of 78 BTM patients. Skandalis et all (2) reported that 7 (\%9) of the 78 BTM diagnosed patients were detected leg ulcer, urticaria, and small vessel vasculitis. In our research, skin disease was detected in all 71 patients with BTM. Higher rates of skin disease in our studies compared to other studies give rise to thought there were a relation with the skin disease rates to be higher the average age of the patients to be high, therefore longer chelation treatment and more UV exposure with increasedmorbidity.

Xerosis is an important psychosocial morbidity condition due to erythema, squamous and itchy developing after atrophy on skin, decreased blood flow, changes in lipid composition ratios and decrease in fluid retention (13). It has been expressed in tissues of BTM that the skin hydration and barrier function associated with iron accumulation increase the risk of developing xerosis $(4,5,13)$. In study of Dogramaci et all, xerosis was reported in $34.6 \%$ of the BTM-diagnosed patients (4). Fahmey SSet all (5) stated that xerosis was determined in the \%22.2 of BTM patients. There was a significant correlation between the incidence of xerosis and high ferritin levels in both studies. Dogramaci et all(4)stated that patients who used deferoxamine and deferiprone had a higher incidence of xerosis than patients using deferasirox; Fahmey et all (5)stated that patients using deferasirox and deferiprone were found to have a higher incidence of xerosis in patients using deferoxamine (4, 5). In our study, the incidence of xerosis in adult BTM patients was determined as $93 \%$. However, there was no significant relationship between xerosis and ferritin levels, chelator treatment agents. According to the other two studies, we found a higher incidence of xerosis in our study; we believe that only adult BTM patients are involved in the study, and therefore the skin structure is deteriorated due to the high average age and the long disease duration, and higher rate of coexistence of comorbid conditions in the study group.
Although iron is an element that plays a role in pigmentation disorders through the formation of hydroxyl radicals and can show toxic effects, it may also have a protective effect on these cells by reducing production of nitric oxide, which is effective in the destruction of melanocytes. Epidermal melanocytes are exposed to the production of highly reactive oxygen species, particularly in cases of inflammation with sun exposure, tanning and postinflammatory hyperpigmentation. Oxidative stress causes malign transformation of melanocytes by disrupting melanocyte homeostasis (14). Dogramaciet all(4) identified pigmentation impairment in $37.2 \%$ of BTM patients and the first order was ephelides $(23.1 \%)$ among the identified pigmentation disorders. Fahmey et all (5)stated that $31.5 \%$ of pediatric BTM patients had pigmentation impairment. In both studies, it was stated that there is a significant relation between the ferritin level and the rate of ephelides incidence. The second most common skin disease in our study was pigmentation disorder with a rate of $77.5 \%$; in the subgroup of pigmentation disorder, ephelides was the most common in $63.4 \%$ and postinflammatory hyperpigmentation in $32.4 \%$ as the second. In our study, Ferritin levels of the patients with ephelide and DBP had more higher than patients without ephelides and DBP.

Acquired nevus are seen so common, with an average of 20 on white race (15). In literature, the prevalence of nevus in BTM patients has not been previously reported. In our study, nevus was detected in $56.3 \%$ of the patients and higher than controls. Age of patients with nevus were more higher than in patients without nevus. The most common melanocytic nevus (52.1\%) was found in the nevus subtype. Melanocytic nevus was detected $\% 63.9$ in males and\%40.0 females. As there is a significant relation between melanocytic nevus incidence rate and male gender.In condition, we think that our study group is related to the presence of adult patients, the long duration of the mean disease increased ferritin levels and medical history and the low rate of UVprotective use associated with high UV exposure due to the geographical location of the region where the study was conducted.

Pruritus is a common complaint in patients with BTM (16) Dogramaci and friends $(\% 37,2)$ ve Fahmey SS et all $(5)$ have determined pruritus(\%37) as the most common skin lesion in BTM patients. This is related to the fact that BTM is a disease that is chronic and requires frequent blood transfusion beside that patients have a xerotic skin (4, 5). In a similar study with both studies, pruritus was detected at $33.8 \%$. Age of patients with pruritus were more higher than in patients without pruritus. We think that pruritus ratio; is related with being a chronic disease that requires drug and frequent blood transfusion in patients with BTM, multiple drug use in patients, increased age, accompanying diseases (xerosisetc).

Dogramaci et all(4) stated that the incidence of dermatosis was $5.4 \%$ in patients with BTM, 3.8\% (most frequent) urticaria, $2.6 \%$ had contact dermatitis, and no significant relationship between serum ferritin and urticaria and contact dermatitis. In a retrospective study, ßthalassemia patients have been reported to develop leg ulcer, cutaneous-small-vessel vasculitis (CSVV) and urticaria at least once in $9 \%$ of patients over a 10 -year period. In this study, it was stated that the most frequently detected is leg ulcer $(0.51 \%)$, except leg ulcer there was a significant relation between high ferritin level and generalized dermatoses. It was stated that may have an inflammatory processenhancing role in the organism of iron overload in ß-thalassemia patients and that the increase in serum ferritin level may be a biologica prognostic marker in generalized dermatoses. Increased serum ferritin levels in patients with generalized dermatosis, indirectly indicate the presence of an increased oxidative burden in the systemic circulation that may cause reversible (urticaria) or irreversible (CSVV) damage in the skin's microvascular system due to localized inflammation (17). In our study; dermatosis was detected in $33.8 \%$ of the patients with major keratosis pilaris in $11.3 \%$ of patients. We think that the risk of developing dermatosis inBTM patients is related with adult age and duration of illness, presence of comorbid conditions, history of multiple drug use, and immune system dysfunction due to increased serum ferritin. The toxic effect of iron overload in macrophages leads to functional disorders in the immune system (2).

In BTM, the risk of infection development increases related to being a disease that is with chronic immune dysfunction and accompanying by multiple comorbidities and during transfusion or chelation treatment associated with recurrent interventional procedures. Dogramaciet all reported that $10.2 \%$ of patients developed skin infection $(5.1 \%$ tinea and $5.1 \%$ verruca) (4). In our study; infective skin lesions (14\% fungal, $8.4 \%$ bacterial and $5.6 \%$ viral) were detected in $26.8 \%$ of the patients. 
There was found a negative correlation between the $\mathrm{Hb}$ value of the patients and tineapedis.In our study, detection of more infectious skin lesions is more likely due to immune function in patients due to the fact that our study group is made up of adults only, and due to more chronic immune dysfunction and comorbid conditions.

Chelator options include deferoxamine, deferasirox, deferiprone, and combination treatments. There are many systemic and dermatological side effects associated with chelator agents $(3,4,18)$. Dogramaci et all (4) reported that $12,8 \%$ of the patients had detected irritation and erythema in the field with deferoxamine pump, xerosis was more common in patients using deferoxamine and deferiprone than deferasirox users. Drug-related skin findings were detected in $21.1 \%$ of the patients treated with chelator treatment. Side effects (atrophic scar, urine and erythema) were most frequently observed with deferoxamine in $11 \%$ of these cases.Ferritin levels of patients with deferoxamine-induced urticaria were more lower than in patients without deferoxamine-induced urticaria. This may be related to the use of deferoxamine more frequently to reduce ferritin levels.

In the literature, there have been no studies researching hair and nail disorders in BTM patients. In our study, it was determined that the patients had the most common telogen efflux $(22.5 \%), 45,1 \%$ had the hair disease and most frequent dystrophic nail $(12,7 \%) ; 35.2 \%$ of the patients had nail disease. There was a statistically significant negative correlation between early whitening and htc levels; between telogen effluum and ferritin levels. All BTM patients with androgenetic alopecia were using hormone replacement treatment agent. Findings of hair and nail found in our study; It may be associated with trace element deficiency and hormone replacement therapy agents, comorbid conditions, and psychosocial morbidity due to chronic progression of BTM, continuous blood transfusion requiringdisease.

As age progresses, collagen and elastin fibers decrease in the dermis. There is also a reduction in total skin thickness as a consequence of flattening the dermal papillae at the epidermal-dermal junction (19). Wollina and Nowak in their studies state that dermatitis and other irritating skin diseases increase with age, and that these increased skin diseases make the drug reaction and other skin diseases easier (20). There was a significant positive correlation between age and IGH, nevus, pruritus and hair diseases in our study. We are of the opinion that the incidence of skin diseases increased due to the age-related deterioration of the skin structure, comorbid diseases and medicines used. We believe that there is no difference in other dermatological diseases due to the similarities of comorbidities, primary diseases and treatment protocols in patients.

In the literature, there were no studies researching dermatological diseases according to genders in patients with BTM. Because of high testosterone levels, male skin is $25 \%$ more thicker than female skin. For this reason, UVA irradiation in women may penetrate better and by causing an increase in iron accumulation in the skin, can cause oxidative damage, which may often affect young women, can give harm to women skin much more (8). In our study, postinflammatory hyperpigmentation and melanocytic nevus in males and telogen effluum in females were found frequently. We believe that this could be explained by psychosocial morbidities are more frequent in women and due to occupational conditions high UV exposure are more frequent in men.

Also, there were no studies reseaching dermatological diseases according to comorbid conditions in BTM patients. Studies have reported increased dermatological diseases in comorbid conditions such as DM, CRF and cardiovascular diseases (21). The relation between chronic diseases such as atherosclerosis and diabetes mellitus and inflammation and oxidative stress has been reported (22). Serum ferritin is suggested as a biomarker for different secondary complications such as diabetes mellitus (23), lower bone mineral density and increased incidence of osteoporosis (19), hypogonadism (24) and generalized xerosis (4) in patients with hereditary hemoglobinopathy. In our study, there was a positive relationship between DM and nevus and androgenetic alopecia in BTM patients. This may be related with increased inflammation and oxidative stress in BTM patients with DM.

\section{CONCLUSION}

We determined that dermatological diseases developed in BTM patients may be related to age, gender, comorbidities, ferritin, $\mathrm{Hb}$ and $\mathrm{Htc}$ levels. A limitation of this study is the small number of patients and control group. Further studies should be conducted with large numbers ofBTM patients and control group.
With large- scale researches, we believe that in BTM patients, the dermatological diseases' relation with oxidative stress is required to be searched and if any relation was found antioxidant usage to prevent dermatological diseases should be taken into consideration. In addition, we believe that the cooperation between the Department of Hematology and Department of Dermatology doctors would be useful for the prevention, early diagnosis and treatment of dermatological diseases that may develop in patients with BTM; thus increasing the quality of life in patients.

\section{Conflict of interest}

No conflict of interest was declared by the authors.

\section{REFERENCES}

1. Modell B, Darlison M. Global epidemiology of haemoglobin disorders and derived service indicators. Bull World Health Organ. 2008;86:4807.

2. Skandalis K, Vlachos C, Pliakou X, Gaitanis G, Kapsali E, Bassukas ID. Higher Serum Ferritin Levels Correlate with an Increased Risk of Cutaneous Morbidity in Adult Patients with $\beta$-Thalassemia: A SingleCenter Retrospective Study. Acta Haematol. 2016;135:124-30.

3.John B. Porter. Optimizing iron chelation strategies in b-thalassaemia major. Blood Reviews 23 Suppl. 2009;1:3-7.

4. Dogramaci AC, Savas N, Ozer B, Duran N. Skin diseases in patients with b-thalassemia major. Int J Dermatol. 2009;48:1057-61.

5. Fahmey SS, Taha G, El-Refaey A, Adly S. Skin Disorders in Egyptian Children with $\beta$-Thalassemia Major. J Trop Pediatr. 2017 May 16.

6.Bomford $A B$, Munro HN. Ferritin gene expression in health and malignancy. Pathobiology. 1992;60:10-8.

7. Baldi A, Lombardi D, Russo P, Palescandolo E, De Luca A, Santini D, et al. Ferritin Contributes to Melanoma Progression by Modulating Cell Growth and Sensitivity to Oxidative Stress. Clin Cancer Res. 2005;11:3175-83.

8. Mansur AT, Aydıngoz IE, Goktay F, Atalay S. Serum Iron and Ferritin Levels in Patients with Vitiligo. Turk derm. 2010;44:153-5.

9. Iuga $A O$, Qureshi $A A$, Lerner EA. Nitric oxide is toxic to melanocytes in vitro. Pigment Cell Res. 2004;17:302-6.

10. Pourzand C, Watkin RD, Brown JE, Tyrrell RM. Ultraviolet A radiation induces immediate release of iron in human primary skin fibroblasts: the role of ferritin. Proc Natl Acad Sci. 1999;96:6751-6.

11. Tisma VS, Basta-Juzbasic A, Jaganjac M, Brcic L, Dobric I, Lipozencic $\mathrm{J}$, et al. Oxidative stres and ferritin expression in the skin of patients with rosacea. J Am Acad Dermatol. 2009;60:270-6.

12. Voskou S, Aslan M, Fanis P, Phylactides M, Kleanthous M. Oxidative stres in $\beta$-thalassaemia and sickle cell disease. Redox Biology. 2015;6:226-39.

13. Farage MA, Miller KW, Berardesca E, Maibach HI. Clinical implications of aging skin: cutaneous disorders in the elderly. Am J Clin Dermatol. 2009;10:73-86.

14. Denat L, Kadekaro AL, Marrot L, Leachman SA, Abdel-Malek ZA. Melanocytes as Instigators and Victims of Oxidative Stress. J Invest Dermatol. 2014;134:1512-8.

15. Burgdorf W.H.C, Plewig G, Wolff H.H. Landthaler M. Braun-Falco's. Dermatology 3rd ed, 2009. SpringerVerlag; 1403-13.

16. Dalgard F, Svensson A, Holm $\lrcorner \varnothing$, et al. Self-reported skin morbidity in Oslo. Associations with sociodemographic factors among adults in a cross-sectional study. Br J Dermatol. 2004;151:452-7.

17. Lotti T, Ghersetich I, Comacchi C. Cutaneous small-vessel vasculitis. J Am Acad Dermatol. 1998;39:667-87.

18. Gulen F, Demir E, Tanac R. Successful desensitization of a case with desferrioxamine hypersensitivity. Minerva Pediatr. 2006;58:571-4.

19. Farage MA, Miller KW, Berardesca E, Maibach HI. Clinical implications of aging skin: cutaneous disorders in the elderly. Am J Clin Dermatol. 2009;10:73-86.

20. Wollina $U$, Nowak A. Dermatology in the intensıve care unit. Our Dermatol Online. 2012;3:298-303.

21. Emre S, Emre C, Akoglu G, Demirseren DD, Metin A. Evaluation of dermatological consultations of patients treated in intensive care unit. Dermatology. 2013;226:75-80.

22. Fulop T, Tessier D, Carpentier A. The metabolic syndrome. Pathol Biol. 54:375-86.

23. Kunutsor SK, Apekey TA, Walley J. Ferritin levels and risk of type 2 diabetes mellitus: an updated systematic review and meta-analysis of prospective evidence. Diabetes Metab Res Rev. 2013;29:308-18.

24. Shalitin S, Carmi D, Weintrob N. Serum ferritin level as a predictor of impaired growth and puberty in thalassemia major patients. Eur J Haematol. 2005;74:93-100. 